\title{
RESEARCH
}

Open Access

\section{Endogenous fluorescence of hemosiderin in endometriosis to improve clinical detection}

\author{
Andrew G. Cabe ${ }^{1,6^{*}} \mathbb{D}$, Arnold D. Estrada ${ }^{3}$, Taylor Hoyt ${ }^{1}$, Xiao Yang ${ }^{1}$, Scott Jenney ${ }^{3}$, Philip T. Valente ${ }^{4}$, Bryan Cox $^{5}$, \\ Jessica E. McLaughlin², Randal D. Robinson², Thomas E. Milner ${ }^{3}$ and Marc D. Feldman ${ }^{1}$
}

\begin{abstract}
Background: Endometriosis impacts 6-10\% of all reproductive- age women. Studies have shown the more effectively endometriosis is removed, the better the patient outcomes for pain reduction and fertility $(2,3)$. Hemosiderin, glands, and stroma are the histologic markers of endometriosis; optical coherence tomography (OCT) can identify glands and hemosiderin has a known endogenous fluorescence than can be detected by two-photon microscopy (TPM). The hypothesis was that the identification of optical properties of endometriosis using OCT and TPM combined would improve a surgeon's ability to diagnose and treat by improving endometriosis detection compared to current standards of visual diagnosis.

Methods: Forty-one women with clinically suspected endometriosis undergoing laparoscopy were consented. Women were enrolled at two clinical sites: University of Texas Health Science Center, San Antonio and Methodist Healthcare System, San Antonio. The surgeon made a clinical diagnosis of suspected endometriosis as 1) yes present 2) maybe present, and 3) not present (controls) from the peritoneum without suspected disease. One-hundred-twenty biopsies were collected from 27 women with visually suspected endometriosis. All three patient biopsy classes were excised and underwent histologic examination as the gold-standard diagnosis for endometriosis. The samples were imaged ex-vivo for optical markers of endometriosis; OCT for endometrial glands and TPM for hemosiderin. Histologic markers were coregistered with optical properties. Biopsies were embedded in agar to maintain orientation during imaging and histological processing. TPM used the endogenous fluorescence of hemosiderin as a marker. OCT used glands as a marker. Sensitivity, specificity, and positive (PPV) and negative predictive values (NPV) were calculated.
\end{abstract}

Results: The main-outcome-measure was the statistical comparison of clinical impression, imaging results, and histologic truth. Glands, stroma and hemosiderin were present in 49, 72 and $86 \%$ of endometriosis samples confirmed by histology. Clinical suspicion of endometriosis had 98\% sensitivity, 53\% specificity, 68\% PPV, and 96\% NPV. In 31 samples of endometriosis maybe being present, 39\% were histologically confirmed. Eighty-eight samples were analyzed using OCTTPM. OCT-TPM had 93\% sensitivity, 100\% specificity, 100\% PPV, and 93\% NPV.

Conclusions: OCT-TPM is useful in identifying endometriosis' presence or absence. Evaluation of suspected endometriosis by OCT-TPM improves surgeons' abilities to diagnose and treat endometriosis.

Keywords: Endometriosis, Optical coherence tomography, Auto-fluorescence

\footnotetext{
* Correspondence: cabe@uthscsa.edu

'Departments of Cardiology, University of Texas Health Science Center at San

Antonio, 7703 Floyd Curl Drive, San Antonio, TX 78229, USA

${ }^{6}$ Department of Medicine, University of Texas Health Science Center, 7703

Floyd Curl Drive, San Antonio, TX 78229, USA

Full list of author information is available at the end of the article
}

(c) The Author(s). 2019 Open Access This article is distributed under the terms of the Creative Commons Attribution 4.0 International License (http://creativecommons.org/licenses/by/4.0/), which permits unrestricted use, distribution, and

reproduction in any medium, provided you give appropriate credit to the original author(s) and the source, provide a link to the Creative Commons license, and indicate if changes were made. The Creative Commons Public Domain Dedication waiver (http://creativecommons.org/publicdomain/zero/1.0/) applies to the data made available in this article, unless otherwise stated. 


\section{Background}

Endometriosis impacts $6-10 \%$ of all reproductive- age women [1]. The gold standard for diagnosis and treatment of endometriosis is laparoscopic inspection and excision or ablation with histologic confirmation. Studies have shown the more effectively endometriosis is removed, the better the patient outcomes for pain reduction and fertility $[2,3]$. However, of the tissue removed during laparoscopy, less than $70 \%$ is confirmed as endometriosis on histologic diagnosis [4]. With a $30-40 \%$ recurrence rate for symptomatic endometriosis after laparoscopic surgery, due to incomplete surgical excision [5], there are clearly short-comings in the current surgical diagnosis and management of endometriosis. During laparoscopic surgery, the surgeon's goal is identification of each endometriosis lesion and sequential excision of each implant. Complicating the time consuming process of identification and removal is the potential widespread distribution of endometriosis lesions throughout the abdominal and pelvic cavities. The distribution of these lesions presents a challenge to all surgeons whose diagnostic imaging tools are normally limited to an unaided eye and laparoscope with some endometriosis not being immediately discernible from surrounding tissue. The nature of the iceberg geometry of endometriosis lesions complicates the surgery since frequently only a fraction of the lesion is visible with the remaining endometriosis lesion located below the surface. Because the surgeon is faced with the divergent challenges of removing all endometrial lesions without damaging delicate underlying tissues and structures, prolonged surgeries are common and only partially successful. Patient outcomes suffer because no effective imaging tools are currently available in the operating room to allow endometriosis lesions to be discerned from surrounding tissues [6].

The current study presents novel solutions for the detection of endometriosis, using optical coherence tomography and two photon microscopy (OCT-TPM) imaging. Hemosiderin is known to have specific excitation/emission wavelengths $[7,8]$. We hypothesized that we could identify hemosiderin as a marker in endometriosis with TPM imaging of the same excitation/emission pair. Hemosiderin is particularly relevant because is present in early-onset endometriosis [9]. Further, it is known that columnar epithelial cells, found in endometriosis glands, form fluid filled glandular structures and OCT is an optical technique capable of easily identifying fluid filled spaces in tissues [10]. Therefore, we hypothesized that OCT could be a second optical tool in the detection of endometriosis. We tested these hypotheses on ex vivo tissue samples collected by gynecologic surgeons from women suspected of having endometriosis. Samples were classified by the surgeon as yes or maybe for the presence of endometriosis, with suspected negative controls also excised, and these samples were then evaluated with both optical techniques and compared to the gold standard, histologic diagnosis. The histologic diagnosis was made by a gynecologic pathologist (PTV) blinded to the results of the OCT-TPM imaging.

\section{Methods}

The tissue samples were collected at two clinical sites, University of Texas Health Science Center at San Antonio, and Methodist Healthcare System in San Antonio. Participants were enrolled between December 18, 2014 and June 6, 2017 in a prospective manner. Two experienced gynecologic surgeons (RDR, BC), each with greater than 25 years' experience diagnosing and treating endometriosis performed all of the surgical procedures.

\section{Ethical approval}

IRB approval for this study was obtained at both clinical sites. Background information was collected on patients' age and race, medical history, and list of medications. Subjects were approached for consent if they were undergoing surgery for suspected endometriosis for clinical indications. Informed consent was obtained for all participants. Subjects were withdrawn if no suspected endometriosis was identified at the time of surgery. The mean participant age was 37 (range 22-46).

\section{Sample collection}

Peritoneal biopsy samples were collected at the time of laparoscopic surgery and the surgeons were asked to make a clinical diagnosis of each sample removed as "yes", "no", or "maybe" for endometriosis. The location of origin of these samples was also recorded. Clinically defined endometriosis was described as red, black, or white lesions. The samples designated in the "no" category were control samples of normal appearing peritoneal tissue biopsied by the surgeon. These three categories allowed us to develop optical signatures of endometriosis. The "maybe" category allowed us to determine if these optical properties could improve the sensitivity and specificity of the surgeon's clinical diagnosis. The "maybe" samples represent suspected disease that some surgeons might remove and others might not depending on location and appearance.

Once the samples were received, imaging was performed to categorize optical properties. Samples were

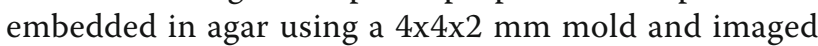
in the mold. The mold allowed for co-registration with histology. Specifically, pre-embedding in a mold allowed the preservation of tissue orientation and location throughout imaging and histological processing. Knowledge of tissue orientation allows exact 3D co-registration between the OCT-TPM images and stained histologic slides. 


\section{Imaging procedures}

Optical imaging was performed with three imaging systems, Optical Coherence Tomography (OCT), Two-Photon Microscopy (TPM), and Wide-Field/Single-Photon Fluorescence. Imaging was performed within $24 \mathrm{~h}$ of sample excision. OCT imaging was performed on a benchtop OCT system built in our lab, using Alazar (Pointe-Claire, QC, Canada) and Santec (Hackensack, NJ, USA) sources, which operate between 1250 and $1376 \mathrm{~nm}$. These systems recorded b-scans for the entire sample volume (up to $6.25 \times 6.25 \times 2 \mathrm{~mm}$ ). Lateral resolution was $6.1-12.21 \mu \mathrm{m} /$ pixel. Axial resolution was $3.75-8 \mu \mathrm{m} /$ pixel. The targets for OCT imaging were glands. The presence of endometriosis detected by OCT was a void in the image which corresponded to a fluid-filled gland in the histology. OCT penetration depth and wide-field also provided a full volume view of the samples, ranging in size from 12 to 27 $\mathrm{mm}^{3}$, useful for later co-registration with histology. The second optical target for endometriosis was the intrinsic fluorescence of hemosiderin. Hemosiderin has an excitation peak of $450 \mathrm{~nm}$, and an emission peak near $680-690$ nm. TPM imaging and Single Photon fluorescence imaging were performed on a Prairie View microscope system (Middleton, WI, USA). TPM was performed using an excitation wavelength of $900 \mathrm{~nm}$ (twice the one-photon excitation peak). Fluorescence images using emission channels, $500 \mathrm{~nm}, 560 \mathrm{~nm}, 620 \mathrm{~nm}$, and $690 \mathrm{~nm}$, were recorded. Hemosiderin was identified by increased pixel intensity in the $690 \mathrm{~nm}$ emission channel. TPM images were taken with a field of view of $735 \mu \mathrm{m}$. Z-series with $10 \mu \mathrm{m}$ step-size were taken and stitched together to image the entire sample. Z-series (stacks of images) were taken in depth until the image was no longer interpretable (approximately $200 \mu \mathrm{m}$ ). TPM had a resolution of $1.44 \mu \mathrm{m} /$ pixel. Additionally, single-photon wide-field fluorescence images were recorded using an excitation source emitting at $420+-20 \mathrm{~nm}$ and an emission filter of $690+-20 \mathrm{~nm}$ to demonstrate hemosiderin detection with standard fluorescence imaging as seen in increased pixel intensity. This method is less expensive than TPM and therefore more translatable to a clinical device.

\section{Histologic processing}

The slides underwent histologic processing. Slides were taken every $40 \mu \mathrm{m}$ for the first $400 \mu \mathrm{m}$ and every $100 \mu \mathrm{m}$ subsequently. Paraffin slides were stained with Hematoxylin and Eosin. For the histologic evaluation, each sample was categorized for the presence of the three histologic markers for endometriosis: glands (columnar endothelial cells), stroma, and hemosiderin. The histologic diagnosis was used as the gold standard against which the surgeons' and imaging systems' findings were compared. Due to the exactness of the match from the use of agar, the optical properties of specific markers were obtained. Unique features in the images were identified as matching the histologic markers.

\section{Statistical analyses}

Sensitivity, specificity and negative and positive predictive values for the accuracy of both the imaging systems and the surgeons' impressions were calculated. Sensitivity was calculated by taking the number of true positives (histologically confirmed endometriosis) and dividing by the sum of the number of true positives and false negatives (histologically confirmed endometriosis that was not detected by the surgeon or imaging systems). Specificity was calculated by taking the number of true negatives (histology confirmed to lack endometriosis) and dividing by the sum of the number of true negatives and false positives (histology confirmed to lack endometriosis but identified as disease positive by the surgeon or imaging system). Positive predictive value was calculated by taking the number of true positives and dividing by the sum of true positives and false positives. Negative predictive value was calculated by taking the number of true negatives and dividing by the sum of true negatives and false negatives.

\section{Results}

OCT was used to identify fluid filled glands and two-photon microscopy (TPM) to detect the endogenous fluorescence of tissue without exogenous labels, specifically hemosiderin. Results show that glands are visible via OCT imaging (Fig. 1a) and hemosiderin has endogenous fluorescence that is present both in multi-photon (Fig. 1b) and single-photon fluorescence imaging (Fig. 1c).

\section{Clinical impression}

This study enrolled 41 women. Based on the clinical diagnosis of the physician, 27 women had endometriosis and 14 did not. These 14 women were determined to have no endometriosis lesions by the surgeon and no biopsies were obtained for ethical reasons. The results outlined below focus on the remaining 27 subjects. Of these 27 subjects, 18 had advanced optical imaging performed with OCT and TPM.

\section{Histology}

For the set of 27 subjects the surgeon's diagnosis of 120 biopsies was compared to the histologic gold standard. The surgeon diagnosed 65 of these as definite endometriosis, 31 as maybe for endometriosis, and 24 as negative for endometriosis. Fifty-seven biopsies were interpreted blindly by the gynecologic pathologist to have endometriosis; 44 of the definite biopsies, 12 of the maybe biopsies and one of the negative biopsies. The accuracy of the surgeons' clinical impression is summarized in Table 1. 

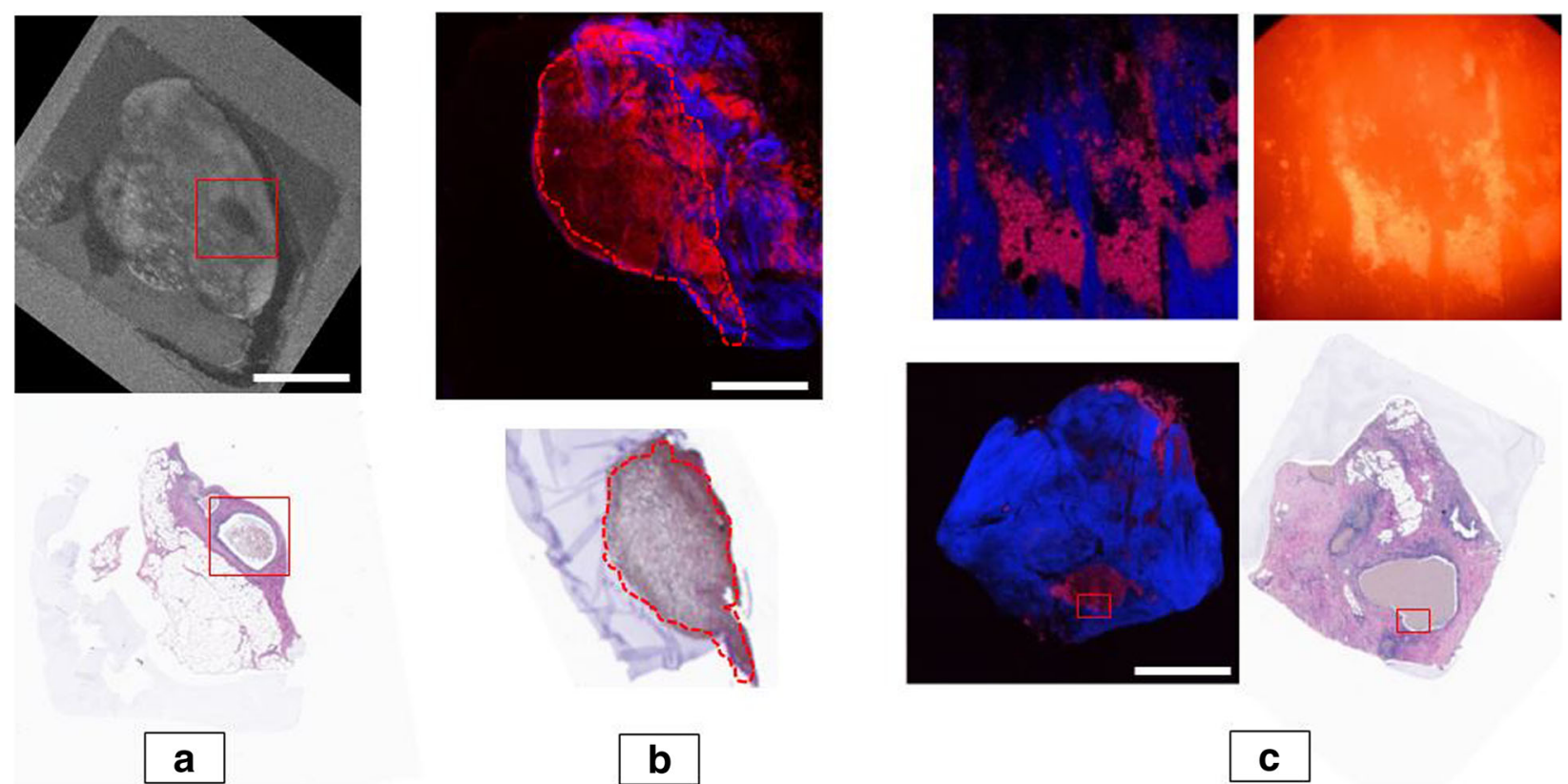

Fig. 1 Imaging Results. 1a Sample 29-1a, the top image is the OCT en face image, the bottom image is the histology, the identified gland is boxed in red, scale bar is $1 \mathrm{~mm}$ 1b Sample 20-6, TPM image on top, histology on bottom, hemosiderin is circled in red and appears as brown in the histology due to H\&E stain, the TPM image is colored based on emission channel, the red represents $690 \mathrm{~nm}$ (hemosideran), blue is $500 \mathrm{~nm}$ (collagen), scale bar is $1 \mathrm{~mm}$ 1c: Sample 34-1 The image on the top left is the single photon microscopy/ wide field fluorescence, very bright spots match with TPM and histology as hemosiderin, the image on the top right is the corresponding TPM image location colored as stated in $1 \mathrm{~b}$ these are scale to $470 \mathrm{um} \times 470 \mathrm{um}$, the bottom right is the full TPM image and bottom left is the corresponding histology, the red box represents the area of the top images, scale bar is $1 \mathrm{~mm}$

Hemosiderin was a prevalent marker seen in 49 of 57 (86\%) endometriosis samples proven positive by histology. Glands were found in pathology in 28 of the 57 (49\%) of endometriosis positive samples. The final marker, stroma, was found in 41 of the 57 samples (72\%), although not an optical target for advanced imaging.

\section{Advanced optical imaging}

It was possible to obtain full optical imaging sets for 18 of the 27 patients outlined above. This resulted in 113 optical imaging sets of co-registered OCT, TPM and histology from 86 biopsy samples. Results are shown in Tables 1, 2, and 3 .

TPM confirmed the presence of hemosiderin with a visible endogenous fluorescence signal in all 36 of those samples where it was confirmed by histology and did not have a single false positive in the other 50 samples (Fig. 2). Glands were visible in OCT for 17 of the 21 samples for which they were seen in histology. OCT for glands lacks a PPV and specificity due to lack of a true false positive. This is due to OCT's optical marker for glands being able to be co-registered with its histologic counterpart, but it is difficult to examine the OCT image and read a false positive that corresponds to histology.

\section{Single photon results}

To allow for clinical translation of hemosiderin as an optical target, 4 subjects were further examined by an additional imaging technique, single-photon fluorescence.

Table 1 Statistical results of surgical impression/ advance optical imaging vs histologic gold standard

\begin{tabular}{|c|c|c|c|c|}
\hline & PPV & NPV & SENS. & SPEC. \\
\hline Surgical Impression (27 subjects) & 68 & 96 & 98 & 53 \\
\hline OCT-TPM Combined (18 subjects with advanced imaging) & 100 & 93 & 93 & 100 \\
\hline TPM for Hemosiderin (113 optical imaging sets) & 100 & 100 & 100 & 100 \\
\hline OCT for Glands (113 optical imaging sets) & & 96 & 82 & \\
\hline
\end{tabular}

PPV is positive predictive value, NPV is negative predictive value, SENS is sensitivity, SPEC is specificity

Surgical Impression is based on the surgical diagnosis of all samples taken from 27 subjects against histological diagnosis. OCT-TPM combined is the optical markers from both imaging type predication of endometriosis against histological diagnosis. OCT-TPM was only available in 18 of 27 patients with endometriosis. One hundred thirteen optical imaging sets were available from these 18 patients for OCT-TPM. OCT for glands lacks a PPV and specificity due to lack of a true false positive. TPM for hemosiderin and OCT for glands is specifically the identification of the marker in the imaging data against histologic confirmation of the marker 
Table 2 Clinical Impression vs. Histology Gold Standard

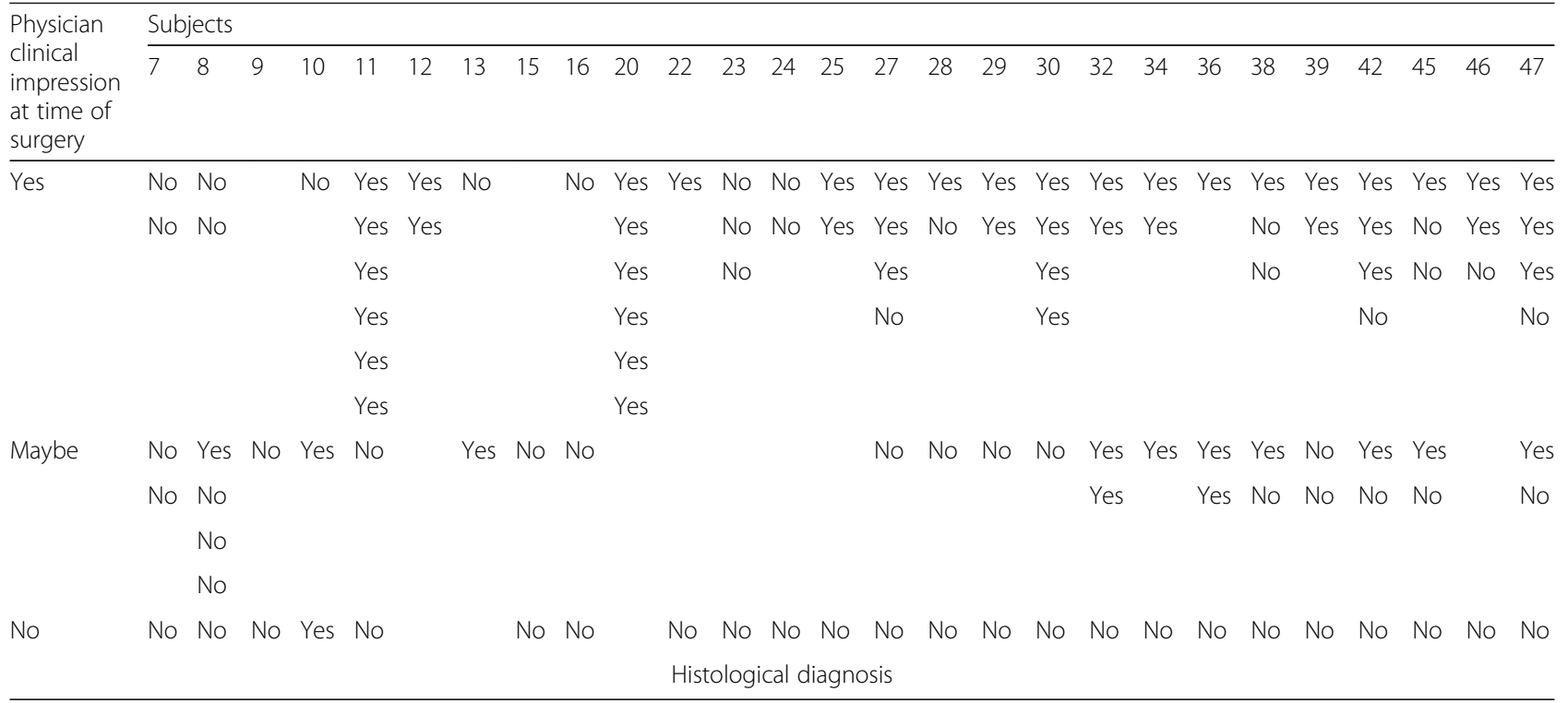

Blank = no sample obtained; each yes or no entry represents each individual sample of tissue biopsied; columns represent the histological diagnosis per the reading of an expert Ob/Gyn pathologist; rows represent Ob/Gyn physician/surgeon clinical impression of the sample at the time of surgery

This evaluation examined 6 biopsies from these 4 patients identified by TPM to be hemosiderin positive, and 2 controls shown by TPM to be hemosiderin negative. Single-photon was able to detect hemosiderin similar to TPM, in the exact areas and identical pattern as TPM, and was confirmed by histology (Fig. 1c).

\section{Discussion}

Our results demonstrate the benefit of adding advanced optical imaging in the diagnosis of endometriosis. The clinician made a correct clinical diagnosis in $67 \%$ of histologic positive samples. Endometrial glands are helpful if present but were only found in histology in 28 of the 57 endometriosis positive samples or $49 \%$ of the time. Glands were visible in OCT for 19 of the $23 \mathrm{im}$ aging sets for which they were seen in histology. The significant finding was the frequency that hemosiderin was present. Hemosiderin was found in $86 \%$ percent of all histologic samples, and correctly identified with TPM $100 \%$ of the time; as well as, in single photon fluorescence imaging, which will allow clinical translation of hemosiderin identification to the operating room.

Specifically, these data demonstrate the need for a better detection method than a surgeon's clinical diagnosis. Of the 65 samples of endometriosis removed where the gynecologic surgeon was certain that endometriosis was present, the surgeon was incorrect 21 times, confirming prior reports of the relatively poor visual diagnosis of endometriosis lesions during laparoscopic surgery for endometriosis. We had two experienced gynecologic surgeons at two institutions and generated 120 total tissue samples for evaluation. Our data is comparable with results from studies with larger cohorts, Mettler reported a $53.8 \%$ positive rate in biopsies of suspected endometriosis [11] and Stratton a $61 \%$ rate [12]. Stegmann reported in her 133 patient, 611 sample study, a true positive rate of $65.0 \%$ rate which is very similar to our $67 \%$ rate but she found a much higher false negative rate of $12 \%$ compared to our $4 \%$ [4].

There are several implications of our results regarding surgeons removing too much tissue in $1 / 3$ of cases and missing lesions that should be removed. If any endometriosis is being missed and left in the patient, the chances for improving patient outcomes of pain reduction and possibly increased fertility, and preventing the high persistence rates common in endometriosis, are diminished. The low specificity and PPV reveals how much non-endometriosis tissue the surgeons are removing during the procedure and how much unproductive surgical time is added to the procedure. The one false negative result reveals that there is diseased tissue that is being left behind because the physician could not determine that a negative biopsy was actually endometriosis. This study also examined "maybe" samples, where the surgical diagnosis was unclear. These samples proved to be positive for endometriosis 39\% of the time. Our combined TPM-OCT imaging accurately classified every "maybe" case. This shows the additive value of an advanced optical imaging approach, which would assist the surgeon in accurately identifying endometriosis where it is unclear whether endometriosis is present or not.

TPM-OCT detection overcomes many of the limitations of other methods developed to improve detection. Studies have shown that specific endometriosis lesions 
Table 3 Full Imaging Results

\begin{tabular}{|c|c|c|c|c|c|c|c|}
\hline Subject & Sample & Subsec. & $\mathrm{Cl}$ & Type & Path. & TPM-hemo & OCT-glands \\
\hline \multirow[t]{9}{*}{20} & 1 & & Yes & Red & $\mathrm{H}$ & Yes & \\
\hline & 2 & & Yes & Red & $\mathrm{H}$ & Yes & \\
\hline & 3 & $b$ & Yes & Red & $\mathrm{H}, \mathrm{S}$ & Yes & \\
\hline & 4 & $b$ & Yes & Dark chocolate cyst & $\mathrm{H}$ & Yes & \\
\hline & & c & Yes & & $S$ & No & \\
\hline & 5 & $a$ & Yes & Cyst & $\mathrm{H}$ & Yes & \\
\hline & & c & Yes & & $\mathrm{H}, \mathrm{S}$ & Yes & \\
\hline & & $d$ & Yes & & $\mathrm{H}$ & Yes & \\
\hline & 6 & & Yes & Endometroma & $\mathrm{H}$ & Yes & \\
\hline \multirow[t]{3}{*}{22} & 1 & $a$ & Yes & Red+black & - & No & \\
\hline & & $b$ & Yes & & $\mathrm{G}, \mathrm{S}$ & No & No \\
\hline & 2 & & No & Control & - & No & \\
\hline \multirow[t]{5}{*}{23} & 1 & & No & Control & - & No & \\
\hline & 2 & a & Yes & Red & - & No & \\
\hline & 3 & a & Yes & red & - & No & \\
\hline & & $b$ & Yes & & - & No & \\
\hline & 4 & $b$ & Yes & Black+white & - & No & \\
\hline \multirow[t]{4}{*}{24} & 1 & a & Yes & Red & - & No & \\
\hline & & $b$ & Yes & & - & No & \\
\hline & 2 & & Yes & Black + white & - & No & \\
\hline & 3 & & No & Control & - & No & \\
\hline \multirow[t]{5}{*}{25} & 1 & a & Yes & Red & S & No & \\
\hline & & $b$ & Yes & & $\mathrm{G}, \mathrm{S}$ & No & No \\
\hline & 2 & $\mathrm{a}$ & Yes & Black & $\mathrm{H}, \mathrm{S}$ & Yes & \\
\hline & & $b$ & Yes & & $\mathrm{H}, \mathrm{S}$ & Yes & \\
\hline & 3 & & No & Control & - & No & \\
\hline \multirow[t]{9}{*}{27} & 1 & a & Yes & Red + black & $G, S$ & No & Yes \\
\hline & & $b$ & Yes & & - & No & \\
\hline & 2 & & Maybe & White & - & No & \\
\hline & 3 & & No & Control & - & No & \\
\hline & 4 & $a$ & Yes & Endometroma & $\mathrm{H}$ & Yes & \\
\hline & & $b$ & Yes & & $\mathrm{H}, \mathrm{S}$ & Yes & \\
\hline & & c & Yes & & $\mathrm{H}$ & Yes & \\
\hline & 5 & & Yes & Black & $\mathrm{H}$ & Yes & \\
\hline & 6 & & Yes & Red & - & No & \\
\hline \multirow[t]{8}{*}{28} & 1 & & Maybe & White & - & No & \\
\hline & 2 & a & Yes & Red & $\mathrm{H}$ & Yes & \\
\hline & & $\mathrm{b}$ & Yes & & - & No & \\
\hline & & c & Yes & & $\mathrm{H}$ & Yes & \\
\hline & 3 & a & Yes & Red & - & No & \\
\hline & & $b$ & Yes & & - & No & \\
\hline & & c & Yes & & - & No & \\
\hline & 4 & & No & Control & - & No & \\
\hline 29 & 1 & a & Yes & Red & $\mathrm{G}, \mathrm{H}, \mathrm{S}$ & Yes & Yes \\
\hline
\end{tabular}


Table 3 Full Imaging Results (Continued)

\begin{tabular}{|c|c|c|c|c|c|c|c|}
\hline Subject & Sample & Subsec. & $\mathrm{Cl}$ & Type & Path. & TPM-hemo & OCT-glands \\
\hline & & $b$ & Yes & & $\mathrm{G}, \mathrm{H}, \mathrm{S}$ & Yes & Yes \\
\hline & 2 & & No & Control & - & No & \\
\hline & 3 & a & Yes & Red + black & - & No & \\
\hline & & $b$ & Yes & & $\mathrm{G}, \mathrm{H}, \mathrm{S}$ & Yes & Yes \\
\hline & 4 & & Maybe & Black & - & No & \\
\hline \multirow[t]{8}{*}{30} & 1 & & Yes & Black & $\mathrm{H}$ & Yes & \\
\hline & 2 & & maybe & White & - & No & \\
\hline & 3 & a & Yes & Black & $\mathrm{H}$ & Yes & \\
\hline & & $b$ & Yes & & $\mathrm{H}$ & Yes & \\
\hline & 4 & & Yes & & $\mathrm{E}, \mathrm{H}$ & Yes & \\
\hline & 5 & a & Yes & & $\mathrm{H}$ & Yes & \\
\hline & & $b$ & Yes & & $\mathrm{E}, \mathrm{H}$ & Yes & \\
\hline & 7 & & No & Control & - & No & \\
\hline \multirow[t]{5}{*}{32} & 1 & & Yes & Red & $\mathrm{G}, \mathrm{H}, \mathrm{S}$ & Yes & Yes \\
\hline & 2 & & Yes & Red & $\mathrm{G}, \mathrm{H}, \mathrm{S}$ & Yes & Yes \\
\hline & 3 & & Maybe & Black & $\mathrm{E}, \mathrm{H}, \mathrm{S}$ & Yes & \\
\hline & 4 & & Maybe & White & $\mathrm{G}, \mathrm{H}, \mathrm{S}$ & Yes & Yes \\
\hline & 5 & & No & Control & - & No & \\
\hline \multirow[t]{5}{*}{34} & 1 & & Yes & Black & $\mathrm{G}, \mathrm{H}, \mathrm{S}$ & Yes & Yes \\
\hline & 2 & a & Yes & Red & $\mathrm{G}, \mathrm{H}, \mathrm{S}$ & Yes & Yes \\
\hline & & $b$ & Yes & & $\mathrm{G}, \mathrm{S}$ & No & Yes \\
\hline & 3 & & Maybe & White & $\mathrm{H}, \mathrm{S}$ & Yes & \\
\hline & 4 & & No & Control & - & No & \\
\hline \multirow[t]{6}{*}{36} & 1 & & No & Control & - & No & \\
\hline & 2 & a & Maybe & Black & $\mathrm{H}, \mathrm{S}$ & Yes & \\
\hline & & $b$ & Maybe & & - & No & \\
\hline & 3 & a & Yes & Red & $\mathrm{G}, \mathrm{S}$ & No & No \\
\hline & & $b$ & Yes & & $\mathrm{E}, \mathrm{H}, \mathrm{S}$ & Yes & \\
\hline & 4 & & Maybe & Black + white & $\mathrm{H}, \mathrm{S}$ & Yes & \\
\hline \multirow[t]{6}{*}{38} & 1 & & Yes & Red & $\mathrm{G}, \mathrm{S}$ & No & Yes \\
\hline & 2 & & Yes & Red & - & No & \\
\hline & 3 & & Yes & Black & - & No & \\
\hline & 4 & & Maybe & White & H & Yes & \\
\hline & 5 & & No & Control & - & No & \\
\hline & 6 & & Maybe & White & - & No & \\
\hline \multirow[t]{8}{*}{39} & 1 & & Yes & & S & No & \\
\hline & 2 & & Maybe & & - & No & \\
\hline & 3 & & No & Control & - & No & \\
\hline & 4 & & Maybe & & - & No & \\
\hline & 5 & a & Yes & & $\mathrm{H}, \mathrm{S}$ & Yes & \\
\hline & & $b$ & Yes & & $\mathrm{H}$ & Yes & \\
\hline & & c & Yes & & - & No & \\
\hline & & $d$ & Yes & & - & No & \\
\hline 42 & 1 & & No & & - & No & \\
\hline
\end{tabular}


Table 3 Full Imaging Results (Continued)

\begin{tabular}{|c|c|c|c|c|c|c|}
\hline Subject & Sample & Subsec. & Type & Path. & TPM-hemo & OCT-glands \\
\hline & 2 & & Maybe & - & No & \\
\hline & 3 & & Yes & - & No & \\
\hline & 4 & a & Maybe & $\mathrm{G}, \mathrm{H}, \mathrm{S}$ & Yes & Yes \\
\hline & & $b$ & Maybe & - & No & \\
\hline & 5 & & Yes & $\mathrm{G}, \mathrm{S}$ & No & Yes \\
\hline & 6 & & Yes & $\mathrm{G}, \mathrm{H}, \mathrm{S}$ & Yes & Yes \\
\hline & 7 & & Yes & $\mathrm{G}, \mathrm{S}$ & No & Yes \\
\hline \multirow[t]{6}{*}{45} & 1 & & Maybe & - & No & \\
\hline & 2 & & Maybe & $\mathrm{H}, \mathrm{S}$ & Yes & \\
\hline & 3 & & Yes & $G, S$ & No & Yes \\
\hline & 4 & & Yes & - & No & \\
\hline & 5 & & Yes & - & No & \\
\hline & 6 & & No & - & No & \\
\hline \multirow[t]{5}{*}{46} & 1 & & Yes & $\mathrm{H}$ & Yes & \\
\hline & 2 & a & Yes & - & No & \\
\hline & & $b$ & Yes & - & No & \\
\hline & 3 & a & Yes & $\mathrm{G}, \mathrm{H}, \mathrm{S}$ & Yes & Yes \\
\hline & 4 & & No & - & No & \\
\hline \multirow[t]{7}{*}{47} & 1 & & Maybe & $\mathrm{G}, \mathrm{S}$ & No & Yes \\
\hline & 2 & & Yes & $\mathrm{G}, \mathrm{H}, \mathrm{S}$ & Yes & No \\
\hline & 3 & & Yes & $\mathrm{G}, \mathrm{H}, \mathrm{S}$ & Yes & Yes \\
\hline & 5 & & Yes & - & No & \\
\hline & 6 & & Maybe & - & No & \\
\hline & 7 & & Yes & $\mathrm{H}, \mathrm{S}$ & Yes & \\
\hline & 8 & & No & - & No & \\
\hline
\end{tabular}

Columns: Subject: Subject number, Sample: sample number from that subject, Subsec.: is the subsection if the sample had to be divided to be fully imaged due to sample size, $\mathrm{Cl}$ (Clinical Impression): yes is positive for endo, maybe is possible endometriosis normally at the surgeon's discretion for removal, no is control tissue, Type: the surgeon reading of type of endometriosis, Path: Pathology reading - $\mathrm{G}$ is gland, fluid filled sac, $\mathrm{E}$ is endothelial cells with no fluid filled sac, $\mathrm{H}$ is hemosiderin, and S is stroma. TPM-hemo: yes is a present optical marker for hemosiderin, no is no optical marker as determined from the TPM image, OCT-glands: yes if the glands is seen and co-registered between the OCT image and histology, no if no such co-registration was possible

are not easily identified laparoscopically or by CT, MRI or ultrasound, unless there are large masses [13, 14]. This limits the surgical relevance of these technologies. Limited success in detecting endometriosis has been seen in comparing blue light adsorption of surrounding tissue with endometriosis, which does not absorb blue light. However blue light techniques do not provide a mechanistic link with a histological gold standard, such as stroma, glands, or hemosiderin and thus its value to clinicians has been questioned and not adopted [15]. Other imaging methods use exogenous materials such as the photo-sensitizer 5-ALA, Firefly ${ }^{\text {max }}$ fluorescence imaging (ICG), and blue dye $[15,16]$. All have had limited success in identifying markers associated with endometriosis, including angiogenesis and enhanced vascularity, changes in the peritoneal tissue surrounding endometriosis, and continued epithelial bleeding. However, the indirect nature of these approaches has also limited widespread adoption. Thus, newer techniques are needed that are both diagnostic based on the true histologic pathology of endometriosis, and marker free as will be shown in a more detailed discussion of these competing optical techniques below.

Demco and co-workers published [17] an endometriosis blue light reflectance/absorption imaging method. The authors hypothesized that lesions not visible under white light were visible under blue light reflectance because porphyrin molecules in endometriosis absorb blue light. This is not an auto fluorescence technique as it looks at absorption of blue light and not emission from excitation. Our TPM detection of hemosiderin looks at the emission signal from excited hemosiderin. Demco's method has not been rigorously tested since there are no sensitivity and specificity results published [17]. Furthermore, the spectral analysis of Demco's technique will not reveal endometriosis below the surface such as 


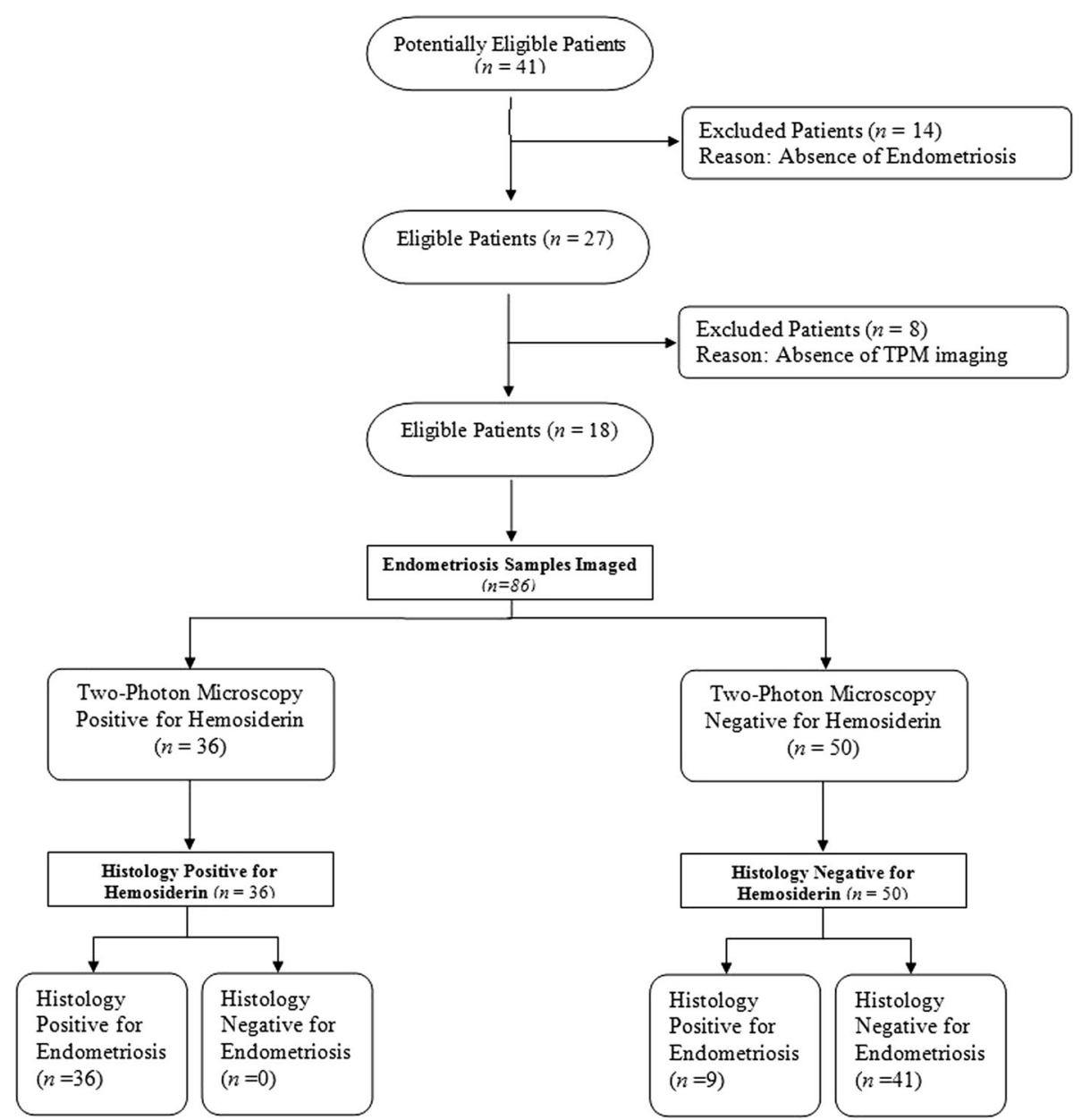

Fig. 2 Endogenous Fluorescence of Hemosiderin to Improve Clinical Detection of Endometriosis. Two-Photon Microscopy (TPM), Histology positive/negative for endometriosis based on pathologist's determination of glands, stroma, and/or hemosiderin

unrecognized intra-ovarian endometriosis, since it relies on surface reflection. Our technique depends on endogenous fluorescence emission and will identify lesions below the surface since we are detecting emitted photons which can pass through layers of tissue.

Buchweitz et al. developed an auto-fluorescence technique to detect differences between normal tissue and endometriosis [18]. They hypothesized that well known auto-fluorescence of NADH, and ATP has a different fluorescence pattern in endometriosis tissue compared to normal tissue. The reasoning behind their approach is that endometriosis has a different metabolic activity than surrounding tissues, and different metabolic rates can be revealed in auto-fluorescence imaging due to the different endogenous fluorescence's of NAD+ and NADH, and ATP and ADP. With Buchweitz's method endometriosis was identified by decreased fluorescence compared to surrounding tissue, looking for a reduction in auto- fluorescence as the marker for endometriosis. The emission wavelengths of those molecules are shorter than the emission wavelength of the porphyrin in hemosiderin, which might explain why they did not see the enhanced fluorescence from hemosiderin as we did $[7,8]$. Looking for a reduction in fluorescence is flawed in that this approach detects a negative image or the absence of a signal.

Another approach utilizes exogenous fluorescence with application of $\delta$-Aminolevulinic acid (5-ALA) which was originally developed for photodynamic therapy in cancer. It has been applied for the detection and treatment of endometriosis. 5-ALA follows the heme pathway and goes into proto-porphyrin IX. They thus are using a similar marker with a similar excitation/ emission as our TPM detection of hemosiderin. However, 5-ALA has several drawbacks. First as an exogenous material it requires a 24-h hospital stay due to its nature as a photo-sensitizer [19]. 5-ALA is a precursor of protoporphyrin IX (PPIX) in the heme pathway. However, PPIX is not in hemosiderin [20], thus 5-ALA targets the increased degree of angiogenesis and vascular endothelial growth factor expression consistent with 
endometriosis. Our techniques present better diagnostic options because they are label free and target physical components of endometriosis rather than metabolic pathways expressed by endometriosis.

Another competing technology is Firefly ${ }^{\text {min }}$ fluorescence imaging which uses indocyanine green (ICG) as an exogenous marker which identifies blood vessels. It has been used in endometriosis detection as it can identify neovascularization associated with endometriosis [21]. Beyond being an exogenous marker, it has several other shortcomings in detection. Specifically, as not all endometriosis presents as 'red' vascularized endometriosis, its ability to accurately detect black and white endometriosis is poor and other methods that do not rely on angiogenesis are needed [22].

A criticism of using TPM to identify hemosiderin is the high cost of the two photon instrument to be used in the operating room. As a result, we evaluated whether the low cost option of single photon fluorescence could be implemented to also identify hemosiderin in endometriosis and found similar accuracy. Clinical translation of these results to a cost-effective device has been built, programed, and its camera and electronic components have been tested. We are currently repeating our studies using this single photon device which can easily be inserted into a laparoscope for clinical translation at low cost. The clinical application of a laparoscope using the specific fluoroscopy detailed in this paper could provide surgeons with the ability to more accurately detect and removed endometriosis.

There are some limitations to our study. Glands and hemosiderin are not present in every endometriosis lesion. Even a TPM imaging system would miss some lesions due to their lack of hemosiderin. However it does present an improved option compared to white light, as TPM has better statistical accuracy. Only 18 of 27 patients identified by the surgeon as having endometriosis had OCT and TPM imaging performed. The clinical study was started before we fully solved the difficult technical issue of co-registration of histology and the region of tissue optically imaged. Embedding the tissue in a cube of agar allowed us to maintain tissue orientation and thus co-registration. Finally, our studies only enrolled a limited number of subjects. Our findings will be expanded on a larger number of patients focusing on single photon in the future. A prospective randomized trial in vivo will have to be completed to ultimately prove the relevance of our findings.

\section{Conclusions}

In summary, focusing on hemosiderin with single photon fluorescence should yield a surgical device capable of improving the detection of endometriosis and allowing clinical translation in a cost effective approach. Both glands seen by OCT and hemosiderin seen by TPM improve a surgeon's ability to correctly diagnose endometriosis. This is demonstrated in improved negative and positive predictive values as compared to the clinical impression of the surgeon. The improved diagnosis by OCT-TPM may result in fewer false positive results and increased disease tissue removed, although this will have to be tested in a prospective clinical trial.

\section{Abbreviations}

5-ALA: $\delta$-Aminolevulinic acid; ICG: Indocyanine green; NPV: Negative predictive values; OCT: Optical coherence tomography; PPIX: Protoporphyrin IX; PPV: and positive; TPM: Two photon microscopy

\section{Acknowledgements}

Not applicable.

\section{Funding}

Clayton Foundation for Biomedical Research, Houston, Texas, USA. The funders had no role in the initiation or design of the study, collection of samples, analysis, interpretation of data, writing of the paper, or the submission for publication.

\section{Availability of data and materials}

The datasets used and/or analysed during the current study not included in this published article are available from the corresponding author on reasonable request.

\section{Authors' contributions}

AGC planned the study, performed all experiments, collected the data, analyzed the results, and wrote the manuscript. ADE helped conduct imaging, devised image processing methods, and contributed to study design. RDR, BC, and JEM preformed surgeries and provided all biopsies. TH and $\mathrm{XY}$ processed imaging data and assisted in image co-registration. SJ contributed to designing image processes and data acquisition. PTV was the expert pathologist and analyzed all histology slides. TEM, RDR, and MDF contributed to study design. ADE, JEM, RDR, TH, TE M, and MDF all assisted in revising the manuscript. All authors read and approved the final version.

Ethics approval and consent to participate

IRB approval was received and maintained at both sites. Informed consent was received from every patient.

Consent for publication

Not applicable.

\section{Competing interests}

The following authors have potential conflicts of interest and have completed International Committee of Medical Journal Editors (ICMJE) forms per their guidelines.

AGC reports personal fees from Clayton Foundation for Research, during the conduct of the study; In addition, AGC has a patent International Patent Application No. PCT/US2018/032877 based on U.S. Serial No. 62/506,910; Entitled "Systems and Methods for Endometrial Tissue Identification" pending to Clayton Foundation for Research.

ADE reports personal fees from Clayton Foundation for Research, during the conduct of the study; In addition, ADE has a patent International Patent Application No. PCT/US2018/032877 based on U.S. Serial No. 62/506,910; Entitled "Systems and Methods for Endometrial Tissue Identification" pending to Clayton Foundation for Research.

TH reports personal fees from Clayton Foundation for Medical Research, during the conduct of the study.

$\mathrm{BC}$ reports personal fees from Clayton Foundation for Research, during the conduct of the study.

RDR reports grants from AbbVie, outside the submitted work.

TEM reports personal fees from Clayton Foundation for Research, during the conduct of the study; In addition, TEM has a patent International Patent Application No. PCT/US2018/032877 based on U.S. Serial No. 62/506,910; 
Entitled "Systems and Methods for Endometrial Tissue Identification" pending to Clayton Foundation for Research.

MDF reports personal fees from Clayton Foundation for Research, during the conduct of the study; In addition, MDF has a patent International Patent Application No. PCT/US2018/032877 based on U.S. Serial No. 62/506,910; Entitled "Systems and Methods for Endometrial Tissue Identification" pending to Clayton Foundation for Research.

All other authors declare that they have no competing interests.

\section{Publisher's Note}

Springer Nature remains neutral with regard to jurisdictional claims in published maps and institutional affiliations.

\section{Author details}

'Departments of Cardiology, University of Texas Health Science Center at San Antonio, 7703 Floyd Curl Drive, San Antonio, TX 78229, USA. ${ }^{2}$ Departments of Obstetrics and Gynecology, University of Texas Health Science Center at San Antonio, 7703 Floyd Curl Drive, San Antonio, TX 78229, USA. ${ }^{3}$ School of Biomedical Engineering, Cockrell School of Engineering, The University of Texas at Austin, 107 W. Dean Keeton, BME Building, 1 University Station C0800, Austin, TX 78712, USA. ${ }^{4}$ Departments of Pathology, University of Texas Health Science Center at San Antonio, 7703 Floyd Curl Drive, San Antonio, TX 78229, USA. ${ }^{5}$ Seven Oaks Women's Center, San Antonio, Seven Oaks Women's Center, 7711 Louis Pasteur, Suite 200, San Antonio, TX 78229, USA. ${ }^{6}$ Department of Medicine, University of Texas Health Science Center, 7703 Floyd Curl Drive, San Antonio, TX 78229, USA.

\section{Received: 14 February 2019 Accepted: 30 April 2019}

Published online: 14 June 2019

\section{References}

1. Jacobson TZ, Duffy JM, Barlow DH, Farquhar C, Koninckx PR, Olive D. Laparoscopic surgery for subfertility associated with endometriosis. In: Jacobson TZ, editor. Cochrane Database of Systematic Reviews. Chichester: Wiley; 2010. https://doi.org/10.1002/14651858.CD001398.pub2.

2. Marcoux S, Maheux R, Bérubé S. Endometriosis the CCG onLaparoscopic surgery in infertile women with minimal or mild endometriosis. N Engl J Med. 1997;337(4):217-22. https://doi.org/10.1056/NEJM199707243370401.

3. Shaw RW. Treatment of endometriosis. Lancet. 1992;340(8830):1267-71. https://doi.org/10.1016/0140-6736(92)92960-N.

4. Stegmann BJ, Sinaii N, Liu S, et al. Using location, color, size, and depth to characterize and identify endometriosis lesions in a cohort of 133 women. Fertil Steril. 2008;89(6):1632-6. https://doi.org/10.1016/j.fertnstert.2007.05.042

5. Abbott JA, Hawe J, Clayton RD, Garry R. The effects and effectiveness of laparoscopic excision of endometriosis: a prospective study with 2 5 year follow-up. Hum Reprod. 2003;18(9):1922-7. https://doi.org/10. 1093/humrep/deg275.

6. Brosens I, Puttemans P, Campo R, Gordts S, Kinkel K. Diagnosis of endometriosis: pelvic endoscopy and imaging techniques. Best Pract Res Clin Obstet Gynaecol. 2004;18(2):285-303. https://doi.org/10.1016/j. bpobgyn.2004.03.002

7. Guy CT, Webster MA, Schaller M, Parsons TJ, Cardiff RD, Muller WJ. Expression of the neu protooncogene in the mammary epithelium of transgenic mice induces metastatic disease. Proc Natl Acad Sci. 1992;89(22): 10578-82. https://doi.org/10.1073/pnas.89.22.10578

8. Perry SW, Burke RM, Brown EB. Two-photon and second harmonic microscopy in clinical and translational Cancer research. Ann Biomed Eng. 2012:40(2):277-91. https://doi.org/10.1007/s10439-012-0512-9.

9. Boppart SA, Goodman A, Libus J, et al. High resolution imaging of endometriosis and ovarian carcinoma with optical coherence tomography: feasibility for laparoscopic-based imaging. BJOG An Int J Obstet Gynaecol. 1999:106(10):1071-7. https://doi.org/10.1111/j.1471-0528.1999.tb08116.

10. Brosens I, Gargett CE, Guo S-W, et al. Origins and progression of adolescent endometriosis. Reprod Sci. 2016;23(10):1282-8. https://doi.org/10.1177/ 1933719116637919.

11. Mettler $L$, Schollmeyer $T$, Lehmann-Willenbrock $E$, et al. Accuracy of laparoscopic diagnosis of endometriosis. JSLS. 2003;7(1):15-8 http://www. ncbi.nlm.nih.gov/pubmed/12722993.

12. Stratton $P$, Winkel C, Premkumar $A$, et al. Diagnostic accuracy of laparoscopy, magnetic resonance imaging, and histopathologic examination for the detection of endometriosis. Fertil Steril. 2003;79(5):1078-85. https:// doi.org/10.1016/S0015-0282(03)00155-9.

13. Stratton P, Winkel CA, Sinaii N, Merino MJ, Zimmer C, Nieman LK. Location, color, size, depth, and volume may predict endometriosis in lesions resected at surgery. In: Fertility and sterility, vol. 78: Elsevier; 2002. p. 743-9. https://doi.org/10.1016/S0015-0282(02)03337-X.

14. Kinkel K, Frei KA, Balleyguier C, Chapron C. Diagnosis of endometriosis with imaging: a review. Eur Radiol. 2006;16(2):285-98. https://doi.org/10.1007/ s00330-005-2882-y.

15. Vlek SL, Lier MCl, Ankersmit M, et al. Laparoscopic imaging techniques in endometriosis therapy: a systematic review. J Minim Invasive Gynecol. 2016; 23(6):886-92. https://doi.org/10.1016/j.jmig.2016.06.019

16. Guan X, Nguyen MTA, Walsh TM, Kelly B. Robotic single-site endometriosis resection using firefly technology. J Minim Invasive Gynecol. 2016;23(1):101. https://doi.org/10.1016/j.jmig.2015.08.001.

17. Demco L. Detection of endometriosis currently depends on the visualization and identification of peritoneal lesions at the time of pelvic laparoscopy. With improved optics, smaller lesions became visible and described. J Am Assoc Gynecol Laparosc. 2004;11(2):2002-5.

18. Buchweitz O, Staebler A, Tio J, Kiesel L. Detection of peritoneal endometriotic lesions by autofluorescence laparoscopy. Am J Obstet Gynecol. 2006;195(4):949-54. https://doi.org/10.1016/j.ajog.2006.02.044.

19. Hillemanns $P$, Weingandt $H$, Stepp $H$, Baumgartner $R$, Xiang W, Korell M Assessment of 5-aminolevulinic acid-induced porphyrin fluorescence in patients with peritoneal endometriosis. Am J Obstet Gynecol. 2000;183(1): 52-7. https://doi.org/10.1067/mob.2000.105897.

20. Beaumont C, Delaby C. Recycling Iron in Normal and pathological states. Semin Hematol. 2009;46(4):328-38. https://doi.org/10.1053/j.seminhematol. 2009.06.004

21. Cosentino F, Vizzielli G, Turco LC, et al. Near-infrared imaging with Indocyanine green for detection of endometriosis lesions (Gre-Endo trial): a pilot study. J Minim Invasive Gynecol. March 2018. https://doi.org/10.1016/J. JMIG.2018.02.023.

22. Laschke MW, Menger MD. In vitro and in vivo approaches to study angiogenesis in the pathophysiology and therapy of endometriosis. Hum Reprod Update. 2007;13(4):331-42. https://doi.org/10.1093/humupd/ dmm006.2016.06.019.
Ready to submit your research? Choose BMC and benefit from:

- fast, convenient online submission

- thorough peer review by experienced researchers in your field

- rapid publication on acceptance

- support for research data, including large and complex data types

- gold Open Access which fosters wider collaboration and increased citations

- maximum visibility for your research: over $100 \mathrm{M}$ website views per year

At BMC, research is always in progress.

Learn more biomedcentral.com/submissions 